EESTI NSV TEADUSTE AKADEEMIA TOIMETISED. XII KOIDE

FOOSIKA-, MATEMAATIKA. JA TEHNIKATEADUSTE SEERIA. 1963, NR. 1

НЗВЕСТНЯ АҚАДЕМИИ НАУК ЭСТОНСКОН ССР. ТОМ ХІІ

СЕРИЯ ФИЗИКО-МАТЕМАТИЧЕСКНХ И ТЕХНИЧЕСКИХ НАУК. 1963. NG I

\title{
ПЕРЕХОДНЫЙ ПРОЦЕСС УПРУГОЙ ДЕФОРМАЦИИ В ЗАМКНУТОЙ КРУГОЦИЛИНДРИЧЕСКОЙ ОБОЛОЧКЕ ПРИ НЕОСЕСИММЕТРИЧЕСКОЙ КРАЕВОЙ НАГРУЗКЕ
}

\author{
Н. АлУмЯЭ, Л. ПОВЕРУС
}

Рассматривается задача об определенин в линейной постановке осевых мембранных напряженнй в полубесконечной замкнутой упругой кругоцилиндрнческой оболочке, возникающих под действием заданных краевых усилий. Закон изменення краевой нагрузки по времени дан единичной функцией Хевисайда, а по дуговой координате $\Phi-$ функцией $\cos n \varphi$, где $n$ - небольшое целое число. За расчетную модель оболоч. ки принята соответствующая уравнениям типа Тимошенко.

Для решения задачи использован метод преобразования Лапласа. Интеграл обращения оценивается численным методом в области непосредственно за фронтом волны и асимптотическими методами в области, достаточно удаленной от фронта волны.

Изучение переходных процессов деформации в тонких. оболочках только начннается; до сего времени в основном внимание было сосредоточено на выяснении тех закономерностей распространения волн по основным формам, которые выражены в графиках фазовой и групповой скоростей и получены на базе уравнений типа Тимошенко (обзор этих работ дан в статье Микловица [1]), а в последнее время также на базе трехмерной теории $\left[{ }^{2}, 3\right]$. Изучению же переходных процессов деформации в пластинках положил начало С. Я. Уфлянд [4]; по этому вопросу имеется ряд работ [5,6] (освещенных также в обзоре [1]); в недавно появившейся статье [ $\left.{ }^{7}\right]$ переходный процесс анализируетея не на модели, а на базе уравнений теории упругости.

Данное исследование о цилиндрической круговой оболочке относится к частному случаю более общего вопроса — могут ли тангенциальные характеристики деформации оболочки при переходном процессе быть определены с помощью безмоментной теории, когда этот процесс вызывается тангенциальными факторами на неасимптотнческом краю оболочки? В рассматриваемом частном случае ответ на этот вопрос будет положительным с оговоркой, что вместо безмоментной нужно пользоваться т. н. полубезмоментной теорией.

1. Основные соотношения. Рассмотрим замкнутую цилиндрическую оболочку вращения, находящуюся под действием краевой нагрузки, которая вызывает в оболочке напряжения и деформации с малым числом ротационной симметрии $n$ ( $n$ - целое число). При этом предположении можно, по-видимому, ряд практических важных задач динамики оболочки решать при помощи упрощенной системы уравнений типа Тимошенко, учитывающих поперечный сдвиг и инерцию вращения только в направлении оси оболочки. * При обычных обозначениях (новым яв-

* Более общие уравнения типа Тимошенко для цилиндрической круговой оболочки, учитывающие поперечный сдвиг и инерцию вращения также в кольцевом направлении, приведены, по-видимому, впервые в статье [8]. 
ляется только $\varphi-$ величина, пропорциональная углу поворота нормали в осевом направлении) для замкнутой цилиндрической оболочки эти уравнения в рассматриваемом случае ( $n-$ малое число) таковы:

$$
\begin{gathered}
u,_{\alpha \alpha}-x^{2} n^{2} u-u, \tau+\left(1-x^{2}\right) n v, \alpha-v w, \alpha=0 \\
-\left(1-x^{2}\right) n u, \alpha+x^{2} v, \alpha-v, \pi-n(n v-w)-\varepsilon^{2} n^{2}(v-n w)=0 \\
v u_{, \alpha}+(n v-w)+x^{2}(w, \alpha \alpha-\varphi, \alpha)-w, \tau+\varepsilon^{2} n^{3}(v-n w)=0 \\
-x^{2}(w, \alpha-\varphi)+\varepsilon^{2}(\varphi, \alpha-\varphi, \tau)=0,
\end{gathered}
$$

где $\alpha, \tau-$ безразмерная координата и время, $x^{2}, \varepsilon^{2}-$ постоянные

$$
x^{2}=(1-v) / 2, \quad \varepsilon^{2}=h^{2} / 12 R^{2}
$$

$v$-- коэффициент Пуассона, $h$ - толщина оболочки, $R$ - радиус ее срединной поверхности; индексы за запятой заменяют символы частных производных по $\alpha, \tau$.

В качестве конкретного примера рассмотрим задачу с краевыми условиями

$$
u,,_{a}+v(n v-w)=H(\tau), \quad v=w=\varphi,{ }_{\alpha} \equiv 0
$$

при $\alpha=0, \tau \geqslant 0$, где $H(\tau)$ - единичная функция Хевисайда: $H(\tau) \equiv 0$ при $\tau<0, H(\tau) \equiv 1$ при $\tau \geqslant 0$. Процесс отражения упругих возмущений от ненагруженного края рассматриваться не будет; таким образом, объектом анализа является т. н. полубесконечная оболочка. Предполагается, что в начале движения оболочка находится в покое; т. е.

$$
u=v=w=\varphi=u, \tau=v, \tau=w_{, \tau}=\varphi_{, \tau}=0
$$

при $\alpha \geqslant 0, \tau=0$. Требуется определить функцию $\sigma(\alpha, \tau)-$ приведенное осевое напряжение

$$
\sigma(\alpha, \tau)=u, \alpha+v(n v-w),
$$

заданное по (1.6) при $\alpha=0: \sigma(0, \tau)=H(\tau)$.

2. Представление решения контурным интегралом. Применение преобразования Лапласа позволяет выразить решение задачи в форме интеграла

$$
\sigma(\alpha, \tau)=\frac{1}{2 i \pi} \int_{c-i \infty}^{c+i \infty} \frac{1}{s} \sum_{k=1}^{4} \sigma_{k}^{*}(s) e^{\alpha r_{k}(s)+\tau s} d s,
$$

где $r_{k}(s)$ - уетыре корня характеристического уравнения

$$
\begin{gathered}
\varepsilon^{2} x^{-4}\left(r^{2}-s^{2}\right)\left(x^{2} r^{2}-s^{2}\right)^{2}+\left(1-v^{2}+s^{2}\right) r^{4}- \\
-\left\{\left[2 n^{2}+3+2 v+\left(1-x^{-2}\right) s^{2}\right] s^{2}+4 \varepsilon^{2} n^{2}\left(n^{2}-1\right)^{2}\right\} r^{2}+ \\
+\left(n^{2}+x^{-2} s^{2}\right)\left(n^{2}+1+s^{2}\right) s^{2}+\varepsilon^{2} n^{4}\left(n^{2}-1\right)^{2}=0,
\end{gathered}
$$

обладающие свойством, что $r(s) / s \rightarrow-1$ при $s \rightarrow \infty$. Пронумеруем эти корни по асимптотическим разложениям 
$r_{1}(s)=-s-\left(n^{2}+v^{2}\right) / 2 s+\ldots, r_{2}(s)=-s / x-x n^{2} / 2 s+\ldots$

$r_{3}(s)=-s-x^{2} / \varepsilon^{2}(1+v) s+\ldots, r_{4}(s)=-s / x+x^{3} / \varepsilon^{2}(1+v) s+\ldots$

За исключением некоторой окрестности точек $s= \pm i \sqrt{1-v^{2}}$ корни уравнения (2.2) достаточно хорошо разделяются, поэтому вне указанной окрестности можно безмоментные (точнее, «полубезмоментные»*) корни $r_{1}(s)$ и $r_{2}(s)$ определить из уравнения

$$
\begin{gathered}
\left(1-v^{2}+s^{2}\right) r^{4}-\left\{\left[2 n^{2}+3+2 v+\left(1-x^{-2}\right) s^{2}\right] s^{2}+\right. \\
\left.+4 \varepsilon^{2} n^{2}\left(n^{2}-1\right)^{2}\right\} r^{2}+\left(n^{2}-x^{-2} s^{2}\right)\left(n^{2}+1+s^{2}\right) s^{2}+ \\
+\varepsilon^{2} n^{4}\left(n^{2}-1\right)^{2}=0,
\end{gathered}
$$

а «моментные корни». $r_{3}(s)$ и $r_{4}(s)$ - из уравнения

$$
\left(r^{2}-s^{2}\right)\left(x^{2} r^{2}-s^{2}\right)+\varepsilon^{-2} \chi^{2}\left(1-v^{2}+s^{2}\right)=0 .
$$

Корнями $\dot{r}_{1}(s), r_{2}(s)$ описывается деформация тангенциального, корнями же $r_{3}(s), r_{4}(s)$ - нормального характера. К тангенциальным факторам относятся, в частности, изображения $\sigma^{*}(\alpha, s)$ и $v^{*}(\alpha, s)$, к нормальным - изображения $w^{*}(\alpha, s)$ и $\varphi, *(\alpha, s)$. Отсюда следует, что вне окрестности точек $s= \pm i \sqrt{1-v^{2}}$ краевые условия могут быть поставлены раздельно; так как на краю $\alpha=0$ задана $\sigma^{*}(0, s)$, то сперва удовлетворяем условиям $\sigma^{*}(0, s)=1 / s, v^{*}(0, s)=0$ напряженными состояниями, соответствующими безмоментным корням $r_{1}(s), r_{2}(s)$, а затем уже остальным условиям $w^{*}(0, s)=0, \varphi,_{a}^{*}(0, s)=0$ при помощи состояний, соответствующих моментным корням $r_{3}(s), r_{4}(s)$. Последние состояния будут при малых значениях $|s|,|s|<\sqrt{1-v^{2}}$ типа краевых эффектов.

По полубезмоментной теории получается следующее выражение для изображения функции $\sigma(\alpha, \tau)$ :

$$
\sigma^{*}(\alpha, s)=s^{-1} \sum_{k=1}^{2} \sigma_{k}^{*}(s) \cdot \exp \left[\alpha r_{k}(s)\right]
$$

где

$$
\sigma_{k}^{*}(s)=\frac{x^{2}\left[v s^{2}+\left(1-v^{2}\right) n^{2}\right] r_{k}^{2}-v\left(s^{2}+x^{2} n^{2}\right)\left[s^{2}-\varepsilon^{2} n^{2}\left(n^{2}-1\right)\right]}{\left(r_{k}^{2}-r_{3-k}^{2}\right)\left[s^{2}+x^{2} n^{2}-(2+v) x^{2} r_{k}^{2}\right]} g(s)
$$

ห

$$
g(s)=\left[1+\left(1+2 v x^{-2}\right) s^{2}\right] \cdot\left(1-v^{2}+s^{2}\right)^{-1} .
$$

3. Напряженное состояние за фронтом упругой волны. Составляющие общего напряженного состояния в некоторой узкой полосе волны $\alpha=\tau$ определяются в основном интеграндами $s^{-1} \sigma_{k}^{*}(s) \cdot \exp \left[\alpha r_{k}(s)+\right.$ $+\tau s]$ в (2.1) при больших значениях $|s|$. Хотя и по (2.3) в этой области независимой переменной $s$ все напряженные состояния имеют качественно одинаковый показатель изменяемости, тем не менее имеет место расчленение напряженных состояний. Чтобы в этом убедиться, достаточно привести головные члены асимптотических разложений по ни-

* Термин «полубезмоментное напряженное состояние» введен В. В. Новожиловым [9]: в книге А. Л. Гольденвейзера $\left[^{10}\right]$ оно названо «выродившимся краевым эффектом». 
сходящнм степеням $s$ величнн $u_{k}^{*}(s), v_{k}^{*}(s), w_{k}^{*}(s), \varphi_{k}^{*}(s)$, нмеющим такое же отношение к $u(\alpha, \tau), v(\alpha, \tau), \quad w(\alpha, \tau), \varphi(\alpha, \tau), \quad$ как $\sigma_{k}^{*}(s)$ в формуле $(2.1)$ к $\sigma(\alpha, \tau)$, например.

$$
u(\alpha, \tau)=\frac{1}{2 i \pi} \int_{c-i \infty}^{c+i \infty} \frac{1}{s} \sum_{k=1}^{4} u_{k}^{*}(s) \cdot e^{\alpha r_{k}(s)+\tau s} d s
$$

Главные члены взанмных отношений прн больших $s \quad\left(\left|s^{2}\right| \gg \varepsilon^{-2}\right)$ следуюшие:

$$
\begin{array}{ll}
v_{1}^{*}=n s^{-1} u_{1}^{*}, \quad w_{1}^{*}=-v s^{-1} u_{1}^{*}, & \varphi_{1}^{*}=-v u_{1}^{*} \\
u_{2}^{*}=n \times s^{-1} v_{2}^{*}, \quad w_{2}^{*}=-(1+v) \chi^{-2} s^{2} n v_{2}^{*}, \quad \varphi_{2}^{*}=2 x n s^{-1} v_{2}^{*} \\
u_{3}^{*}=v \varepsilon^{2} \varphi_{3}^{*}, \quad v_{3}^{*}=v n \varepsilon^{2} s^{-1} \varphi_{3}^{*}, & w_{3}^{*}=2(1+v)^{-1} \chi^{2} s^{-1} \varphi_{3}^{*} \\
u_{4}^{*}=-2 v(1+v)^{-1} x s^{-1} w_{4}^{*}, & v_{4}^{*}=(1+v) x^{-2} \varepsilon^{2} n w_{4}^{*} \\
& \varphi_{4}^{*}=-2(1+v)^{-1} \chi^{3} \varepsilon^{-2} s^{-1} w_{4}^{*},
\end{array}
$$

откуда следует, что корни $r_{1}(s), r_{2}(s)$ описывают тангенциальную деформацию, корни же $r_{3}(s), r_{4}(s)$ - нормальную. ния

В области, где $\varepsilon^{0} \ll|\operatorname{Res}|^{2} \ll \varepsilon^{-2}$, получим вместо (3.1) соотноше-

$$
\begin{aligned}
& v_{1}^{*}=n s^{-1} u_{1}^{*}, \quad w_{1}^{*}=-v s^{-1} u_{1}^{*}, \quad \varphi_{1}^{*}=-v u_{1}^{*} \\
& u_{2}^{*}=n \varkappa s^{-1} v_{2}^{*}, \quad w_{2}^{*}=2 x^{2} n s^{-2} v_{2}^{*}, \quad \varphi_{2}^{*}=2 x^{2} n s^{-1} v_{2}^{*} \\
& u_{j}^{*}=-v \varepsilon^{2} r_{j}\left(r_{j}^{2} / s^{2}-x^{-2}\right) w_{j}^{*} \\
& v_{j}^{*}=-n \varepsilon^{2} \chi^{-2}\left[\left(2+v+v^{2}\right) r_{j}^{2} / 2 s^{2}-1\right] w_{j}^{*} \\
& \varphi_{j}^{*}=r_{j} w_{j}^{*} \quad(j=3,4),
\end{aligned}
$$

тде $r_{3}(s), r_{4}(s)$ - корни уравнения (2.5): Эти соотношения опять-таки показывают расчленение общего напряженного состояния на безмоментное и моментное состояния в рассматриваемой области изменения $s$. Вместе с тем, для определения изображения функции $\sigma(\alpha, \tau)$ В правой полуплоскости $s,(\operatorname{Re} s \gg 0)$ применима безмоментная теория и формулы (2.6), (2.7).

Предложено несколько методов для численного обращения преобразования Лапласа. Один из них будет типа

$$
\sigma(\alpha, \tau)=\sum_{j=1}^{m} H_{j}^{(m)} \sigma^{*}\left(\alpha, \eta_{j}^{(m)} / \tau\right)
$$

где $H_{j}^{(m)}, \quad \eta_{j}^{(m)}$ - табулированные комплексно-сопряженные числа $\left[{ }^{11,12}\right]$, причем. точки $s_{j}^{(m)}=\eta_{j}^{(m)} / \tau$ находятся на правой полуплоскости $s$; другие - типа

$$
\sigma(\alpha, \tau)=\sum_{j=1}^{m} A_{j}^{(m)}(\tau) \cdot \sigma^{*}\left(\alpha, s_{j}\right)
$$


использующие значения $\sigma^{*}\left(\alpha, s_{j}\right)$ в некоторой последовательности точек $s_{j}$ на положительной полуоси $s\left[{ }^{13,14}\right]$. Формулы, приведенные в статьях $[11-13]$, дают точный ответ, когда интегранд в интеграле обращения является полиномом относительно $1 / s$. Отметим, что варианты, требующие определения $\sigma^{*}(\alpha, s)$ только в точках положительной полуоси $s$, имеют некоторое преимущество, так как в этом случае число действий с комплексными величинами относительно мало.

Для иллюстрации поведения функции $\sigma(\alpha, \tau)$ за фронтом волны $\alpha=\tau$ вдоль прямых $\alpha=\alpha_{m}=m \pi / 2(m=1,2,3,4)$ указанные выше алгоритмы расчета не использовались, так как изображение $\sigma^{*}\left(\alpha_{m}, s\right)$ функций $\sigma(\alpha, \tau)$ оказалось при $m=3$ и $m=4$ плохо аппроксимируемым при помощи полиномов (отыскивалось приближение в смысле функции Чебышева $\sigma^{*}\left(\alpha_{m}, s\right)$, заданной в 14 точках $s_{p}$ в интервале $0 \leqslant 1 / s_{p}<1$ положительной полуоси $s$ ); также неудачно окончились попытки применить методы, описанные в $\left[{ }^{14}\right]$. K удовлетворительному же приближению $\sigma^{*}\left(\alpha_{m}, s\right)$ в интервале $0<1 / s<1$ привела рациональная аппроксимация B форме *

$$
\widehat{\sigma}_{m}\left(\alpha_{m i}, s\right) \approx \frac{1}{s^{2}} \frac{k_{0 m}+k_{1 m} s+k_{2 m} s^{2}+s^{3}}{k_{3 m}+k_{4 m} s+s^{2}},
$$

допускающее обращение в элементарных функциях. Коэффициенты $k_{\text {jm }}$ определялись симплекс-методом линейного программирования из условия

$$
\sum_{p=1}^{13} u_{p m}=\min
$$

где

$$
\begin{gathered}
u_{p m}=1+s_{p}^{-1} k_{2 m}+s_{p}^{-2} k_{1 m}+s_{p}^{-3} k_{0 m}- \\
-s_{p} \widehat{\sigma}_{m}\left(\alpha_{m}, s_{p}\right) \cdot\left[1+s_{p}^{-1} k_{3 m}+s_{p}^{-2} k_{4 m}\right] \geqslant 0,
\end{gathered}
$$

a $s_{p}$ - точки на положительной полуоси $s$.

\section{Обращением (3.3) будет функция}

$$
\sigma\left(\alpha_{m}, \tau_{m}\right)=c_{0 m}-c_{1 m} \tau_{m}+e^{-\lambda m^{\tau} m}\left(c_{2 m} \cos \omega_{m} \tau_{m}+c_{3 m} \sin \omega_{m} \tau_{m}\right) ;
$$

соотношения между коэффициентами $c_{0 m}, \ldots, c_{3 m}, \lambda_{m}, \omega_{m}$ и $k_{0 m}, \ldots$, $k_{4 m}$ ради краткости изложения не приводятся; их вывод элементарен.

Численные результаты даны в табл. 1 для случая $n=2$ ( $n-$ число ротационной симметрии напряженного состояния).

Таблица 1

\begin{tabular}{c|c|c|c|c|c|c}
\hline$m$ & $c_{0 m}$ & $c_{1 m}$ & $c_{2 m}$ & $c_{3 m}$ & $\lambda_{m}$ & $\omega_{m}$ \\
\hline 1 & 0,436 & 0,084 & 0,564 & $-0,834$ & 2,10 & 2,35 \\
2 & 0,094 & 0,052 & 0,906 & $-0,835$ & 2,92 & 3,97 \\
3 & 0,066 & 0,036 & 0,934 & $-1,093$ & 3,54 & 5,11 \\
4 & 0,066 & 0,028 & 0,934 & $-1,196$ & 4,39 & 6,50
\end{tabular}

* Временно предполагается, что преобразование Лапласа проведено не по времени $\tau$, а по $\tau_{m}$, где $\tau_{m}=\tau-\alpha_{m} ;$ нзображение по $\tau_{m}$ обозначается через $\widehat{\sigma}\left(a_{m}, s\right)$. 
Таблица отражает тот известный факт, что с увеличением $\alpha$ показатели изменяемости функции $\sigma(\alpha, \tau)$ по $\tau$ за фронтом волны $\alpha=\tau$ всё увеличиваются.

4. Асимптотические свойства $\sigma(\alpha, \tau)$ при больших значениях $\tau$. Методы определения решения при больших значениях $\tau$ связаны с деформированием контура интегрирования, что требует выяснения аналитических свойств интегранда в (2.1).

Число точек разветвления корней $r_{k}(s)$ уравнения $(2.2)$ весьма большое - 24, из которых в полуплоскости $\operatorname{Re} s>0$ имеется 6 ; тем не менее интеғранд (но не его отдельные слагаемые $\sigma_{k}{ }^{*} \exp \alpha r_{k}$ ) регулярен при $\operatorname{Re} s>0$. Для цальнейшего существенными являются, во-первых, точки разветвления корней на мнимой оси. плоскости $s$, где один из корней обрашается в нуль; это точки

$$
\begin{gathered}
s_{1}=i \varepsilon n\left(n^{2}-1\right)\left(n^{2}+1\right)^{-1 / 2}, \quad s_{3}=i\left(n^{2}+1\right)^{1 / 2} \\
s_{4}=i x n, \quad s_{5}=i x / \varepsilon,
\end{gathered}
$$

во-вторых, точка разветвления около $s=i \sqrt{1-v^{2}}$ :

$$
s_{2}=i \sqrt{1-v^{2}}+3 \cdot 2^{-3 / 3} \varepsilon^{2 / 3}\left(1-v^{2}\right)^{1 / 6}\left(2 n^{2}+v^{2}\right)^{2 / 8} e^{-5 i \pi / 6} ;
$$

разветвление происходит также в точках $\bar{s}_{h}$ комплексно сопряженных точкам $s_{h}$ в $(4.1),(4.2), h=1,2, \ldots, 5$. Отсюда следует, что для однозначного определения интегранда в (2.1) достаточно провести 10 срезов от $-\infty$ до точек $s_{h}, \bar{s}_{h}$, параллельных вещественной оси плоскости $S$.

Асимптотические формулы для $\sigma(\alpha, \tau)$ при больших значениях $\tau$ и малых $\alpha / \tau$ получаются наиболее просто деформированием контура интегрирования в (2.1) так, что контур идет вдоль берегов каждого из вынеуказанных срезов. Если назвать обход каждой особой точки $s_{h}$ $\left(\bar{s}_{h}\right)$ субконтуром $c_{h}\left(\bar{c}_{h}\right)$, то этих субконтуров будет всего десять, образующих пять комплексно-сопряженных пар. Интегрирование по двум комплексно-сопряженным субконтурам дает в сумме одну вещественную функцию; таким образом, получается пять функций $\sigma^{(i)}(\alpha, \tau)$. Решение можно представить в следующей форме:

$$
\sigma(\alpha, \tau)=\sum_{h=1}^{5} \sigma^{(h)}(\alpha, \tau)+\sigma^{(0)}(\alpha, \tau)
$$

где $\sigma^{(0)}(\alpha, \tau)$ - результат обхода полюса при $s=0$; при $n=2, v=0,3$ имеем

$$
\sigma^{(0)}(\alpha, \tau)=\exp [-2,51 \sqrt{\varepsilon} \alpha] \cdot \cos 2,51 \sqrt{\varepsilon} \alpha .
$$

$\sum_{h=1}^{5} \sigma^{(h)}$ Пписывает переходный процесс. .

С увеличением отношения $\alpha / \tau$ щелесообразно провести контур интегрирования через седловые точки функций $\operatorname{Re}\left[r_{k}(s)+s \tau / \alpha\right]$; это позволяет получить асимптотические формулы при $\tau \rightarrow \infty$ методом перевала. При $\alpha / \tau \gtrsim \varepsilon^{1 / 2}$ контуры интегрирования $c_{1}, \overline{c_{1}}$ оставят полюс $s=0$ слева от себя, поэтому в секторе $\alpha / \tau \geqslant \varepsilon^{1 / 2}$ решение получается в форме 


$$
\sigma(\alpha, \tau)=\sum_{h=1}^{5} \sigma^{(h)}(\alpha, \tau)
$$

где при $\tau \rightarrow \infty$

$$
\sigma^{(h)}(\alpha, \tau)=\frac{1}{\sqrt{\tau}} a_{h}(\alpha / \tau) \cdot \cos \left[\tau p_{h}(\alpha / \tau)+b_{h}\right]
$$

Исключением является случай, когда седловые точки двух смежных контуров сливаются; в этом случае соответствующие колебания затухают медленнее - вместо коэффициента $\tau^{-1 / 2}$ получается $\tau^{-1 / 3}$.

Приводим в качестве иллюстрации некоторые числовые результаты, относящиеся к случаю $n=2, v=0,3$.

$1^{\circ}$. При малом отношении $\alpha / \tau\left(\alpha \ll \varepsilon^{1 / 2} \tau\right)$ и $\tau \sim \varepsilon^{-1}$ имеем

$$
\sigma^{(1)}(\alpha, \tau)=\frac{\alpha}{\sqrt{\varepsilon \tau}} \varphi(\tau)
$$

где $\uparrow(\tau)$ представлена табл. 2.

\begin{tabular}{c|c|c|c|c|c|c|c}
$\boldsymbol{\varepsilon} \tau$ & 0,124 & 0,186 & 0,249 & 0,372 & 0,558 & 0,744 & 1,116 \\
\hline$\varphi(\tau)$ & 0,138 & 0,147 & 0,151 & 0,149 & 0,120 & 0,061 & $-0,079$
\end{tabular}

Отметим, что при $\tau>\varepsilon^{-1 / 2}$ необходимо применение полубезмоментной теории вместо более простой безмоментной теории. Данные в табл. 2 получеңы вычислением интеграла обращения по шеститочечной формуле интегрирования гауссова типа. Асимптотика функции $\varphi(\tau)$ при $\tau \rightarrow \infty$ начинает вырисовываться лишь при весьма больших значениях $\tau\left(\tau \gg \varepsilon^{-3}\right)$, поэтому соответствующая асимптотическая формула практического интереса не представляет.

Для области, где $\alpha / \tau \gg \varepsilon^{1 / 2}$, можно получить при помощи безмоментной теории и методом перевала асимптотическую формулу типа (4.6). Коэффициенты $a_{1}(\alpha / \tau), p_{1}(\alpha / \tau)$ даны в табл. $3, b_{1}=-3 \pi / 4$.

\begin{tabular}{c|c|c|c|c|c|c}
$\alpha / \tau$ & 0,208 & 0,233 & 0,252 & 0,264 & 0,272 & 0,276 \\
\hline$a_{1}(\alpha / \tau)$ & 2,25 & 1,84 & 1,69 & 1,65 & 1,74 & 2,16 \\
\hline$p_{1}(\alpha / \tau)$ & 0,057 & 0,071 & 0,083 & 0,095 & 0,101 & 0,107
\end{tabular}

Как следует из этой таблицы, амплитуда $a_{1}(\alpha, \tau)$ волны с увеличением отношения $\alpha / \tau$ растет, начиная с $\alpha / \tau=0,264$. Әто результат того, что при $\alpha / \tau=0,280$ две смежные седловые точки сливаются в одну. Вдоль луча $\alpha / \tau=0,280$ получим следующее асимптотическое уравнение:

$$
\sigma^{(1)}+\sigma^{(2)}=-0,522 \tau^{-1 / 3} \sin 0,107 \tau \text {. }
$$

$2^{\circ}$. Составляющая волны напряжения $\sigma^{(2)}(\alpha, \tau)$, которая опреде ляется интегралами по субконтурам $c_{2}, \overline{c_{2}}$, может быть при немалых значениях $\alpha / \tau$ определена также при помощи безмоментной теории методом перевала. Результаты вычисления коэффициентов $a_{2}(\alpha / \tau), p_{2}(\alpha / \tau)$ формулы (4.6) при $b_{2}=-\pi / 4$ даны в табл. 4. 
Таблица 4

\begin{tabular}{l|c|c|c|c|c|c|c|c|c}
$\alpha / \tau$ & 0,276 & 0,265 & 0,253 & 0,235 & 0,214 & 0,191 & 0,165 & 0,135 & 0,109 \\
\hline$a_{2}(\alpha / \tau)$ & 1,09 & 0,64 & 0,40 & 0,26 & 0,17 & 0,11 & 0,065 & 0,034 & 0,016 \\
\hline$p_{2}(\alpha / \tau)$ & 0,106 & 0,089 & 0,068 & 0,032 & 0,013 & 0,069 & 0,138 & 0,228 & 0,316
\end{tabular}

С дальнейшим уменышением отношения $\alpha / \tau$ амплитуда $a_{2}(\alpha / \tau)$ все убывает; при $\alpha / \tau \sim \varepsilon^{3 / 4}$ можно еще с погрешностью порядка $\sqrt{\varepsilon}$ по сравнению с единицей пользоваться безмоментной теорией, что с применением метода перевала дает

$$
\sigma^{(2)}(\alpha, \tau)=0,094(\alpha / \tau)^{1 / 3} \tau^{-1 / 2} \cos (0,954 \tau+\pi / 4) .
$$

Если же $\alpha / \tau \leqslant \varepsilon^{3 / 4}$, то перевалы на контурах будут находиться близко к точкам $s= \pm i \sqrt{1-v^{2}}$, где безмоментные и моментные напряженные состояния не расчленяются. Применение моментной теории в частном случае $\alpha=15,5 \varepsilon \tau$ (по-видимому, при этом-значении $\alpha / \tau$ функция $a_{2}(\alpha / \tau)$ имеет локальный минимум) дает

$$
\begin{gathered}
\sigma^{(2)}=\varepsilon^{1 / 3} \tau^{-1 / 2}\{0,26 \cos (0,954 \tau+\pi / 4)- \\
-0,03 \sin (0,954 \tau+\pi / 4)
\end{gathered}
$$

впрочем, подставляя в формулу (4.9) это частное значение $\alpha / \tau$, получим коэффициент 0,23 вместо 0,26 . Это показывает, что безмоментная теория дает при малых $\alpha / \tau$ по сравнению с моментной теорией несколько заниженные значения амплитуд волны.

Локальный максимум волны $\sigma^{(2)}(\alpha, \tau)$ при малом $\alpha / \tau$ достигается при $\alpha=8,04 \varepsilon \tau$ :

$$
\sigma^{(2)}=-1,05 \varepsilon^{4 / 9} \tau^{-1 / s} \sin 0,954 \tau ;
$$

вдоль этого луча колебания затухают относительно медленно, как и в случае, представленном формулой (4.8). При дальнейшем убывании отношения $\alpha / \tau$ волна $\sigma^{(2)}$ будет затухатьь уже по экспоненциальному закону.

$3^{\circ}$. Обход контурами интегрирования $c_{3}, \bar{c}_{3}$ особых точек $s_{3}, \bar{s}_{3}$ определяет волну $\sigma^{(3)}(\alpha, \tau)$, которая может быть определена по безмоментной теории. Полученные методом перевала коэффициенты формулы (4.6) при $b_{3}=3 \pi / 4$ представлены в табл. 5.

таблица 5

\begin{tabular}{l|l|l|l|l|l|l|l|l|l|l|l|l|}
$\dot{\boldsymbol{\alpha}} / \tau$ & 0,260 & 0,342 & 0,373 & 0,389 & 0,400 & 0,410 & 0,422 & 0,434 & 0,447 & 0,459 & $0,470 \mid$ & 0,481 \\
\hline$a_{3}(\alpha / \tau)$ & 0,398 & 0,688 & 0,944 & 1,108 & 1,070 & 0,914 & 0,764 & 0,648 & 0,564 & 0,500 & 0,452 & 0,413 \\
\hline$p_{3}(\alpha / \tau)$ & 1,113 & 1,049 & 1,015 & 0,992 & 0,973 & 0,953 & 0,927 & 0,898 & 0,865 & 0,830 & 0,794 & 0,759
\end{tabular}

$4^{\circ}$. Соответствующие данные для волны $\sigma^{(4)}(\alpha, \tau)$ приведены в табл. 6, которые опять-таки вычислены по безмоментной теории.

На рис 1 для сравнения представлены амплитудные функции $a_{h}(\alpha / \tau)$ безмоментных волн $\sigma^{(h)}(\alpha, \tau)$. 
таблица 6

\begin{tabular}{l|l|l|l|l|l|l|l|l|l|l}
$\alpha / \tau$ & 0,204 & 0,318 & 0,418 & 0,492 & 0,550 & 0,599 & 0,639 & 0,673 & 0,702 & 0,727 \\
\hline$a_{4}(\alpha / \tau)$ & 0,0564 & 0,0889 & 0,1114 & 0,1424 & 0,1718 & 0,2010 & 0,229 & 0,256 & 0,284 & 0,312 \\
\hline$p_{4}(\alpha / \tau)$ & 2,45 & 2,71 & 3,02 & 3,32 & 3,61 & 3,89 & 4,17 & 4,44 & 4,70 & 4,96
\end{tabular}

По безмоментной теории не может быть определена слагаемая $\sigma^{(5)}(\alpha, \tau)$, получаемая как результат интегрирования по субконтурам, обходящим особые точки $s_{5}, \bar{s}_{5}$. Однако особенности в этих точках имеют момегтные корни характеристического уравнения (2.2), поэтому амплитуды-соответствуюших волн $\sigma^{(5)}(\alpha, \tau)$ весьма малы.

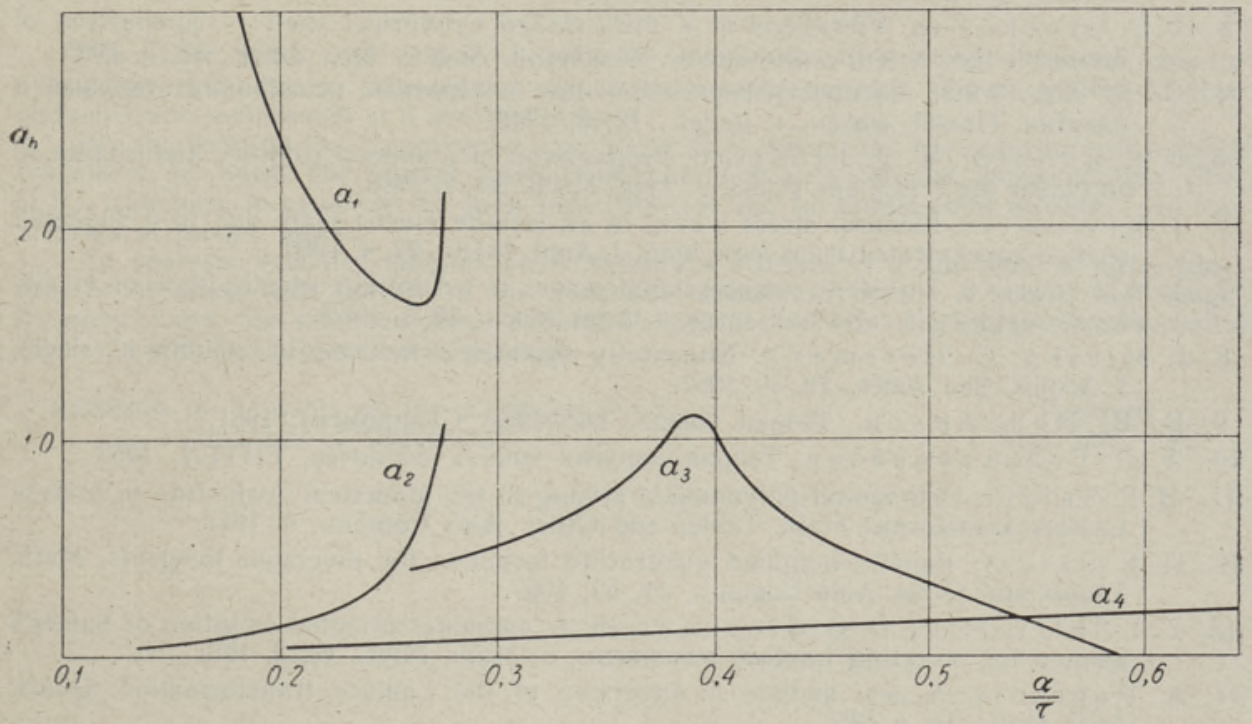

Рнс. 1.

Во всех остальных случаях можно для определения $\sigma^{(h)}(\alpha, \tau)$ пользоваться безмоментной теорией. В самом деле, амплитуда волны $\sigma^{(2)}(\alpha, \tau)$ в интервале $\alpha / \tau$, где нужно вместо безмоментной применять моментную теорию, по формуле (4.11) весьма мала по сравнению с $\sigma^{(0)}(\alpha, \tau)$ - установившейся частью функции $\sigma(\alpha, \tau)$, которая определена через (4.4).

Замена полубезмоментной теории безмоментной кажется возможной только при оболочках коротких или же, в крайнем случае, при оболочках средней приведенной длины, если число ротационной симметрии $n$ невелико.

Таким образом, полубезмоментная теория определяет с достаточной точностью осевые напряжения $\sigma(\alpha, \tau)$.

Совершенно естественно возникает вопрос -- является ли этот вывод частным случаем или же можно величины тангенциального характера определить по безмоментной (полубезмоментной) теории, когда переходный процесс вызывается тангенциальными факторами на неасимпто- 
тическом краю произвольной оболочки? Решение этого общего вопроса требует дальнейшего исследования. Если длительность рассматриваемого процесса небольшая и выполнены условия применимости безмоментной теории при статической задаче, то ответ на этот вопрос будет, по-видимому, положительным.

\title{
ЛИТЕРАТУРА
}

1. J. Miklowitz, Recent developments in elastic wave propagation, Appl. Mech. Rev.. $13,12,1960$.

2. D. C. G a z is, Three-dimensional investigation of the propagation of waves in hollow circular cylinders, J. Acoust. Soc. Amer., 31, 5, 1959.

3. J. E. Greenspon, Vibrations of a thick-walled cylindrical shell - comparison of the exact theory with approximate theories, J. Acoust. Soc. Amer., 32, 5, 1960.

4. С. Я. У флянд, Распространение волн при поперечных колебаниях стержней и пластин, Прикл. матем. и механ., 12, 3, 1948.

5. J. N. Goodier, W. E. J a h s ma n, Propagation of a sudden rotatory disturbance in an elastic plate in plane stress, J. Appl. Mech., 23, 2, 1956.

6. J. Miklow itz, Flexural stress waves in an infinite elastic plate due to a suddenly applied concentrated transverse load, J. Appl. Mech., 27, 4, 1960,

7. J. Miklowitz, Transient compressional waves in an infinite elastic plate or elastic layer overlying a rigid half-space, J. Appl. Mech., 29, 1, 1962.

8. I. Mirsky, G. Herrmann, Nonaxially symmetric motions of cylindrical shells. J. Acoust. Soc. Amer., 29, 10, 1957.

9. В. В. Новожилов, Теория тонких оболочек, Судпромгиз, 1951.

10. А. Л. Гольденвей зер, Теория упругих тонких оболочек, ГИТтЛ, 1953.

11. H. E. S a lzer, Orthogonal polynomials arising in the numerical evaluation of inverse Laplace transforms, Math. Tables and Other Aids Comput., 9, 1955.

12. H. E. S a I zer, Equally-weighted quadrature formulas for inversion integrals, Math. Tables and Other Aids Comput., 11, 59, 1957.

13. C. I. Shirtcliffe, D. G. Stephenson, A computer oriented adaption of Salzer's method for inverting Laplace transforms, J. Math. Phys., 40, 2, 1961.

14. A. P a poulis, A new method of inversion of the Laplace transformation, Quart. Appl. Math., 14, 4, 1957.

Ннститут кибернетики

Академии наук Эстонской ССP
Поступила в редакцию

14. VIII 1962

\section{SULETUD RINGSILINDRILISE KOORIKU OTSA JÄRSUL MITTETELGSOMMEETRILISEL. KOORMAMISEL ARENEVA ELASTSE DEFORMATSIOONI OLEMINEKUPROTSESSIST}

\author{
N. Alumäe, L. Poverus
}

\section{Resümee}

Artiklis käsitletakse elastse kooriku membraanjõudude määramist lineaarsete Timo. šenko-tüüpi vōrrandite abil. Lōpliku suurusega koormis - teljesuunalised membraan jōud - rakendatakse silindri otsale järsku ja säilitatakse; koormise jaotus paralleelringi koordinaadi $\varphi$ järgi on $\cos n \varphi(n-$ väike täisarv $)$. 
Olesande lahendamiseks kasutatakse Laplace'i teisendusmeetodit. Inversiooniintegraal arvutatakse koormamise algetapil teisenduse ratsionaalse aproksimatsiooni (3.3) abil.

Artiklis näidatakse, et membraanjōudude määramiseks ōhukeseseinalises silindris on rakendatav üldise momentide teooria asemel märksa lihtsam semimembraaniteooria, koormamise algetapil aga isegi membraaniteooria.

\author{
Eesti NSV Teaduste Akadeemia Sabus toimetusse \\ Küberneetika Instituut \\ 14. VIII 1962
}

\title{
TRANSIENT STRESSES IN A SEMI-INFINITE ELASTIC CYLINDRICAL SHELL UNDER NONAXIAL LOADING
}

\author{
N. Alumäe, L. Poverus
}

- Summary

This paper deals with a determination of membrane stresses in a closed circular cylindrical shell due to sinusoidally distributed membrane edge forces, applied in time suddenly and maintained at a constant vaiue.

For the solution the Tymoshenko-type linear shell theory and the procedure of Laplace transform are used. The inverse integral is evaluated by a rational approximation (3.3) of the transform to establish the early-time behavior and by saddle-point method - to find stresses at longer times.

An analysis indicates that transient membrane stresses in a thin shell at early times may be obtained with the aid of the dynamical membrane theory of shells; for longer transient times the semimembrane dynamical theory of shells (including circumferential moments and shear forces) must be used.

Academy of Sciencés of the Estonian S.S.R.,

Received Institute of Cybernetics 